\title{
Sitting Position in Neurosurgery
}

\author{
Shwetal Goraksha ${ }^{1}$ Bhoomika Thakore ${ }^{1} \quad$ Joseph Monteiro $^{1}$
}

\author{
${ }^{1}$ Department of Anesthesiology, P.D. Hinduja Hospital and Medical \\ Research Centre, Mumbai, Maharashtra, India
}

\begin{abstract}
Address for correspondence Joseph Monteiro, MD, Department of Anesthesiology, P.D. Hinduja Hospital and Medical Research Centre, D 61, Nirvana, Bhagoji Keer Marg, Mahim, Mumbai 400016, Maharashtra, India (e-mail: monteiro04@gmail.com).
\end{abstract}

\begin{abstract}
Neurosurgery in the sitting position is used for resection of tumors located in the cerebellopontine angle, pineal region, other technically difficult tumors in the infratentorial region, as well as surgeries of the cervical spine. Since its inception, the use

Keywords

- patent foramen ovale

- sitting position

- venous air embolism of this particular position has been contentious due its risk-benefit analysis. With improved microsurgical equipment and surgical and anesthetic techniques, as well as advanced monitoring techniques to assure patient safety, many centers are revisiting the sitting position for its potential benefits.
\end{abstract}

\section{Introduction}

The sitting position, anatomically and physiologically, has several advantages such as good surgical access, less bleeding, and better ventilation. However, it could be associated with potentially devastating complications, such as venous air embolism (VAE), cerebral ischemia due to hemodynamics compromise, quadriplegia, tension pneumocephalus, and compressive neuropathy. ${ }^{1}$ Historically, any kind of surgical intervention in the posterior fossa of the brain was avoided since it was believed that any type of manipulation in this area could lead to loss of respiration, sudden deterioration, and even death. ${ }^{2}$ In 1906, Sir Victor Horsley performed the first posterior fossa surgery in the lateral oblique position. ${ }^{3}$ The first neurosurgical procedure in the sitting position was performed by De Martel in 1913, for a posterior fossa tumor under local anesthesia. ${ }^{4}$ In 1928 in the United States, Frazier and Gardner used this position for surgery on the Gasserian ganglion to treat trigeminal neuralgia. ${ }^{5}$ Slbin et al in 1976 published their experience of 180 neurosurgical patients in this position. ${ }^{6}$ Since this position has numerous possible complications, many neurosurgeons may prefer the prone position. However, the advantages of sitting or semisitting position over prone are many. In the sitting position, there is improved access to the tracheal tube, face, chest wall, and arms. Once the patient is positioned prone, the access to the airway is very difficult, not to mention that the endotracheal tube is vulnerable to movement and tube position needs to be rechecked clinically repeatedly, to rule out endobronchial intubation or accidental extubation. Pressure exerted on the abdomen and raised intra-abdominal pressure may decrease thoracic compliance and increase airway pressure, which in turn can increase intracranial pressure (ICP). Numerous potential pressure ulcer areas in the prone patient include the face, breasts, genitalia, and bony prominences. Postoperative facial edema, visual loss, and retinal ischemia as a result of direct pressure on the eye has also been reported following prone surgery. ${ }^{7}$

\section{Indications}

Sitting position is used for resection of tumors located in the cerebellopontine angle, pineal region, other technically difficult tumors in the infratentorial region, as well as surgeries of the cervical spine. ${ }^{8}$ The surgical advantage over lateral or prone position for similar surgeries is that the brain is relaxed because the blood and cerebrospinal fluid (CSF) are drained by gravity, and the tumor separates from the cranial nerves without much retraction, and improves operator orientation and access to deep midline posterior fossa structures, while allowing unobstructed view of the face for cranial nerve monitoring and ease of resuscitation in the event of catastrophe. ${ }^{3}$ However, we need to keep in mind patient safety while deciding the best position for surgical access. ${ }^{6}$

\section{Preoperative Optimization}

To ensure patient safety and a successful outcome, a thorough preoperative anesthetic evaluation to decide if the sitting position is suitable for the patient is essential. As in any neurosurgical case, all patients need to be evaluated preoperatively for physical and neurological status, as well
DOI https://doi.org/ 10.1055/s-0039-1693078 ISSN 2348-0548.
Copyright @2020 Indian Society of Neuroanaesthesiology and Critical Care
License terms

() (1) $\ominus \circledast$ 
as blood and other pertinent investigations to formulate an appropriate anesthetic plan. Cardiovascular assessment (electrocardiogram [ECG], echocardiogram) is important because there may be perioperative blood pressure fluctuations, ECG changes, arrhythmias, and myocardial ischemia or failure, which occur due to central neurogenic effects on the myocardium and the autonomic nervous system or concurrently associated medical conditions. Any signs such as dysphagia, loss of gag reflex, and altered state of consciousness should be noted preoperatively as these are important for assessment at emergence and to plan postoperative care. Documented preoperative ability of the patient to move the neck without neurological symptoms such as paraesthesia, pain, or dizziness should be ascertained. This precludes the use of the sitting position in affected patients, so as to prevent unnecessary or excessive movement of the neck and any further impairment of function that may occur due to positioning during surgery.

\section{Contraindications}

In the sitting position, the risk of venous air embolism (VAE) is high. It may have cardiovascular, pulmonary, and neurological sequelae. After analysis of 28 studies conducted from 1972 to 2007, Fathi et al found the incidence VAE to be 0 to $76 \%$ in sitting position as compared to 0 to $12 \%$ in the horizontal position. ${ }^{9}$ The presence of a patent foramen ovale (PFO), which is about $25 \%$ in the normal population, is a contraindication to performing any surgery in the sitting position as it can lead to paradoxical air embolism (PAE) with resultant ischemia or infarction in the heart or brain through the right-to-left shunt. In the above meta-analysis by Fathi et al, the incidence of paradoxical embolism in neurosurgical procedures in the semisitting position was between 0 and 14\%. ${ }^{9}$ In 2018, Klein et al in their systematic review analyzed four observational studies with a total of 977 patients who underwent surgery of the posterior fossa or cervical spine in the semisitting position, of whom 82 had a PFO, and observed an incidence of VAE in 33 of these 82 patients (40.2\%) and found no incidence of paradoxical embolism. They also surmised that if the PFO is large or if there is a permanent right-to-left shunt with history of PAE, closure of PFO, usually transcutaneously, should be preferably done prior to surgery. PFO can be detected preoperatively by two main methods: Transesophageal echocardiography (TEE) allows for direct visualization of the interatrial septum and PFO, whereas a transcranial Doppler (TCD) is a highly sensitive indirect method relying on microbubbles detected in a cerebral artery (middle cerebral artery [MCA]) after the intravenous injection of air in the presence of a PFO. ${ }^{10}$ Thus, a mandatory contrast echo/bubble test done preoperatively could help rule out a PFO, if the sitting position is to be used.

Other relative contraindications for neurosurgery in the sitting position include atherosclerotic cardiovascular disease, hemodynamic instability, and severe cervical canal stenosis. ${ }^{11}$

\section{Anesthesia Technique and Monitoring}

Neuroanesthesia requires a good understanding of neurophysiology to maintain adequate cerebral hemodynamics while providing optimum operating conditions. Induction with intravenous agents such as propofol, opioids, and nondepolarizing muscle relaxants attenuates the hypertensive response to laryngoscopy and intubation, with minimal effects on intracerebral hemodynamics. For neurosurgery in the sitting position, reinforced endotracheal tubes are preferred to prevent intraoperative kinking, which may lead to increase in the airway pressure and ICP. The endotracheal tube should also be carefully secured ensuring that there is no obstruction of cerebral venous drainage. ${ }^{12}$ Although there are no consensus recommendations, total intravenous anesthesia (TIVA) is usually preferred. In a meta-analysis by Chui et al, both propofol and volatile anesthesia had similar brain relaxation scores; however, mean ICP values were lower and CPP values higher with propofol. ${ }^{13}$ Bastola et al in their study of 75 patients concluded that propofol, sevoflurane, and desflurane had comparable clinical profiles when used for craniotomies. ${ }^{14}$

Nitrous oxide, however, should be avoided as it causes cerebral vasodilation and increase in cerebral blood flow (CBF) and ICP. Since it has a high blood:gas partition coefficient (0.47), the use of nitrous oxide could worsen intraoperative VAE, if it occurs, and any existing postoperative pneumocephalus. Losasso et al in their study have repudiated this finding and found that $50 \%$ nitrous oxide did not increase the risk of VAE. ${ }^{15}$ In another study by Losasso et $\mathrm{al}$, the use of nitrous oxide helped reduce opioid and inhalational agent requirement, facilitating early recovery and neurologic assessment. ${ }^{16}$

The use of PEEP (positive end-expiratory pressure) during neurosurgical procedures performed in the sitting position is another controversy. Physiological PEEP of 4 to $5 \mathrm{~cm} \mathrm{H}_{2} \mathrm{O}$ helps decrease the venous gradient as the increase in the transthoracic pressure is transmitted to the central veins, which increases the CVP. This helps decrease the amount of air entrained during VAE. Voorhies et al recommended PEEP for both prevention and treatment of VAE, ${ }^{17}$ whereas Giebler et al concluded that PEEP in this position should be abandoned as it does not decrease the incidence of VAE but is associated with significant adverse cardiovascular effects. ${ }^{18}$ Dilmen et al used PEEP at physiological levels $\left(5-10 \mathrm{~cm} \mathrm{H}_{2} \mathrm{O}\right.$ ) to prevent VAE. ${ }^{19}$

In addition to the routine monitoring of the neurosurgical patient, specific monitoring is required to screen for known complications such as VAE. Capnography (end-tidal $\left.\mathrm{CO}_{2}\left[\mathrm{ETCO}_{2}\right]\right)$ is used as a standard of care monitor for all cases of general anesthesia, and a sudden drop of $2 \mathrm{~mm} \mathrm{Hg}$ or more in the $\mathrm{ETCO}_{2}$ is highly suggestive of air embolism. Historically, the esophageal stethoscope was used to confirm a Mill-Wheel murmur that is classically associated with large intracardiac air emboli and described as a "characteristic splashing auscultatory sound." However, the sensitivity of the esophageal stethoscope is very low. The change in the heart sounds are not detected until the rate of infusion of air 
is more than $1.7 \mathrm{~mL} / \mathrm{kg} / \mathrm{min} .{ }^{20}$ Precordial Doppler is highly sensitive as compared to capnography for the diagnosis of VAE. However, it is a subjective, nonquantitative method, and the anesthesiologist needs to be familiar with it use. ${ }^{21}$ TEE has the advantage of making a precise diagnosis and is very sensitive. TEE allows for the detection of small air volumes in the heart, even $0.01 \mathrm{~mL} / \mathrm{kg}$ of air. Its use is essential for diagnosing the patent foramen. ${ }^{9}$ Feigl et al evaluated the risk of PAE in patients with PFO, and concluded that with standardized anesthesia, TEE, and capnometry monitoring for VAE, these patients can be safely operated in the semisitting position. ${ }^{22}$ Pandia et al, in a trial with 140 patients undergoing surgery of the posterior fossa, made a comparison between using $\mathrm{ETCO}_{2}$ and TEE for the diagnosis of air embolism and concluded that in the presence of a diagnosis of air embolism using TEE with no associated changes in capnography, it is unlikely for the air embolism to cause any hemodynamic imbalances. ${ }^{23}$

Another monitor that has recently emerged to further ensure patient safety in the sitting position is regional cerebral oximetry $\left(\mathrm{rSO}_{2}\right)$. Heroabadi et al in 2017 found that there was a statistically significant reduction in mean arterial pressure (MAP) and $\mathrm{rSO}_{2}, 15$ minutes after establishment of sitting position, but this was not clinically significant and may not increase the risk of cerebral hypoxia in elective neurosurgery in the sitting position. ${ }^{24}$ Schramm et al in 2016 studied cerebral oxygen saturation in supine and sitting positions at predetermined endpoints. They found that $\mathrm{rSO}_{2}$ values increased after sitting position, and therefore it was safe to use this position for cerebral oxygen saturation. ${ }^{25}$

\section{Technique of Positioning}

Positioning of the neurosurgical patient in the sitting position is challenging. It is the responsibility of both the surgeon and anesthesiologist. Patient positioning is done after induction of general anesthesia and placement of arterial and venous lines, and requires adequate anesthesia depth and maintenance of hemodynamic stability. ${ }^{26}$

The sitting or Fowler's position is a standard patient position in which the patient is seated in an upright sitting position (45-60 degrees) and may have knees either bent or straight. In the semisitting position, the head elevation is less, about 30 to 45 degrees, and the foot of the bed is raised at the knee to bend the legs. In the modified sitting position, the table is positioned in a steep Trendelenburg position, the back end is elevated, and the Mayfield clamp is fixed at the back section of the table. In case of VAE, by lowering the back section of the table, it can be returned to the horizontal position. The lower extremities are semiflexed with legs resting on pillows, thus reducing flexion and kinking of blood vessels of the lower extremities and therefore improving venous return and decreasing the risk of thromboembolism. ${ }^{27}$

\section{Complications}

In this position, the incidence of hypotension varies from $5 \%$ to $32 \%$. This is due to pooling of blood in the lower limbs, decreasing the venous return, stroke volume, and cardiac output. The resultant hypotension decreases cerebral perfusion pressure (СРP) and can result in cerebral hypoxia. ${ }^{28}$ Anesthetic agents, both inhalational and intravenous such as propofol, have vasodilator properties and depress cardiac contractility that may also contribute to the hemodynamic instability. Preoperative intravascular status and positive-pressure ventilation further aggravate the hypotension. Therefore, the patient should be preloaded with adequate intravenous fluids and the sitting position gradually given over a period of 5 to 10 minutes with continuous monitoring of blood pressure, to check whether the patient tolerates the same. Ranjith et al, in their prospective observational trial of 20 patients, preloaded the patients with a volume of $20 \mathrm{~mL} / \mathrm{kg}$ of crystalloid before positioning them from supine to sitting position. This maintained hemodynamic stability and avoided any vasopressor requirement. ${ }^{29}$

Dohn and Gardner in 1956 devised a " $G$ " suit that consisted of inflatable leggings and an abdominal binder, which, by constricting the lower part of the body, prevented pooling of blood and increased the venous return, and thus reduced hypotension in anesthetized patients in this position. ${ }^{30}$ Meyer et al studied the use of antishock trousers (MAST suit) and PEEP 8 to $10 \mathrm{~cm} \mathrm{H}_{2} \mathrm{O}$ in children undergoing neurosurgical procedures in the sitting position. After the sitting position was given, inflation of the MAST suit induced a dramatic increase in right atrial pressure (RAP) and jugular bulb venous pressure (JBVP), reinforced by addition of PEEP. ${ }^{31}$ However, ventilation and hypoperfusion of intra-abdominal organs need to be borne in mind. Intermittent pneumatic compression stockings for the lower limbs should therefore be mandatorily used for all patients who are operated in the sitting position. However, as noted by Martin, these have minimal value as prophylaxis against air embolism. ${ }^{32}$ Correct positioning of the patient with flexion of the hips and elevation of the knees to the level of the heart will also minimize the hypotension; modified sitting position is therefore advocated. ${ }^{3}$ Dilmen et al found the incidence of hypotension with positioning to be $37.6 \%$ in adults and $18.6 \%$ in children, as well as a correlation between high ASA (American Society of Anesthesiologists) physical status and hypotension with positioning. ${ }^{19}$

Extreme flexion of the head and neck may obstruct the jugular venous and lymphatic drainage, which increases the cerebral venous pressure and may cause postoperative face, tongue, soft palate, and posterior pharyngeal wall edema. This can lead to postoperative airway obstruction, hypoxemia, and need for reintubation and prolonged ventilation. Use of oral airways and long duration of surgery are also associated with this condition. ${ }^{3,33}$ Rath et al noted that infants are at a higher risk of edema because of a small tracheal diameter and relatively large tongue. ${ }^{34}$

Cervical spine ischemia with neck and head hyperflexion, especially compounded by hypotension, can lead to quadriplegia. The risk increases with age and vascular and structural cervical spine pathology. It is recommended to keep a sufficient distance of at least two to three fingerbreadths between the chin and nearest bone, usually the clavicle. ${ }^{3}$ Somatosensory evoked potential monitoring has been proposed as an indicator of the adequacy of regional spinal cord 
perfusion in these cases. Buchheit et al in their study in 1998 concluded that SSEPs may help identify, and thus prevent, midcervical flexion myelopathy secondary to placement of a patient in the semisitting or sitting position..$^{35}$

Incidence of peripheral nerve injuries is less than $1 \%$. As per the "Summary of Task Force Consensus on the Prevention of Perioperative Peripheral Neuropathies Relevant to Positioning for Neurosurgery," padding at the elbow decreases the risk of upper extremity neuropathy. Specific padding is used to prevent pressure of a hard surface against the peroneal nerve at the fibular head to decrease the risk of peroneal neuropathy. ${ }^{36}$

The main concerns in the sitting position are the complications that can occur and their sequelae as noted previously. VAE is a potentially serious complication that occurs since the site of surgery is above the level of the heart, which causes a negative pressure or subatmospheric gradient between the right atrium and the cranial venous sinuses, and allows the air to entrain into the venous system. Pathophysiology and clinical manifestations of VAE depend on amount and rate of air entrainment. ${ }^{37}$ The lethal amount of air entrained for adults is 3 to $5 \mathrm{~mL} / \mathrm{kg}$. Once the air gets into the circulation, it passes through the right atrium into the pulmonary circulation and impedes gas exchange that causes the drop in $\mathrm{ETCO}_{2}$. When the amount of air is small, the pulmonary capillaries can filter it, but when the air volume is larger, there may be significant right ventricular outflow obstruction with decrease in cardiac output, hypotension, and myocardial and cerebral ischemia. Modest amounts of air entrainment into the pulmonary circulation may lead to pulmonary vasoconstriction, release of inflammatory mediators, bronchoconstriction, and an increase in ventilation-perfusion mismatch. If the embolism is large ( $>5 \mathrm{~mL} / \mathrm{kg}$ ), a gas airlock scenario can lead to right-sided heart failure and immediate cardiovascular collapse. ${ }^{21}$ The true incidence of VAE depends on the method of detection used. Ganslandt et al in 2013 in their study of 600 cases had an overall 19\% incidence of VAE. The incidence of VAE differed depending on the monitoring technique used for detection of VAE. The incidence was $25.6 \%$ of the cases in which TEE was used, and $9.4 \%$ of the patients monitored with Doppler ultrasound. ${ }^{38}$ Fathi et al found that the incidence of VAE ranged from $25 \%$ to $50 \%$ as documented through precordial Doppler and up to $76 \%$ with TEE. ${ }^{9}$ Mammoto et al studied 21 patients undergoing neurosurgery in the sitting position and monitored those patients with TEE and capnography. During VAE, microbubbles detected on TEE in the right atrium were noted, and the severity was graded as follows: grade 0 ( 0 microbubble), grade $1(<5$ microbubbles per frame), grade 2 (10-12 microbubbles per frame), and grade 3 (too many microbubbles to be counted per frame). A fall in $\mathrm{ETCO}_{2}$ greater than 3 to $4 \mathrm{~mm} \mathrm{Hg}$ occurred with every episode of grades 2 and 3 air embolus. ${ }^{39}$ Feigl et al used the Tubingen scale to grade VAE: grade 0 (no air bubbles visible, no air embolism); grade I (air bubbles on TEE); grade II (air bubbles on TEE with decrease of ETCO $\leq 3 \mathrm{~mm} \mathrm{Hg}$ ); grade III (air bubbles on TEE with decrease of ETCO2 $>3 \mathrm{~mm} \mathrm{Hg}$; grade IV (air bubbles on TEE with decrease of ETCO2 $>3 \mathrm{~mm} \mathrm{Hg}$ and decrease of mean arterial pressure $\geq 20 \%$ or increase of heart rate $\geq 40 \%$ (or both); and grade V (VAE causing arrhythmia with hemodynamic instability requiring cardiopulmonary resuscitation). ${ }^{22}$ Harrison et al noted that VAE can occur at any time during the surgery and noted an incidence of VAE of 9.3\% (38 of 407 of pediatric neurosurgery cases). These occurred during opening of the dura, tumor dissection, and on closure of the dura. ${ }^{40}$ The treatment for VAE is aimed at stopping further entrainment of air into the circulation and managing any complications. If VAE is suspected either by fall in $\mathrm{ETCO}_{2}$ or microbubbles on TEE, the neurosurgeon is immediately informed and the surgical field should be irrigated or covered with saline soaked swabs, ventilated with $100 \%$ oxygen and intravenous fluids, and, if necessary, vasopressors started to support the circulation. ${ }^{21,37,40}$ Transient bilateral compression of the jugular veins is a technique described to reduce the inflow of air through the exposed sinuses, as well as identify the source of air entrainment. In neurosurgical procedures performed in the sitting position, when VAE occurs, transient manual jugular venous compression limits further air entry into the circulation. As per the study by Eckle et al, an intrajugular balloon was placed into bilateral jugular vessels and inflated during VAE, as an alternative technique aimed at reducing the risk of air embolism. Additionally, accumulated air could be aspirated from an orifice of the catheter. These findings may help in further development of intrajugular balloon catheters designed to reduce the risk of air embolism. However, two central venous lines would have to be placed, and temporary inflation and compression of both veins could damage the intimal layer of the vessel. Additional risks include accidental arterial puncture or cannulation, nerve injury, and pneumothorax. ${ }^{41}$

Longatti et al in their preliminary study of 10 cases suggested that a $\mathrm{CO}_{2}$-enriched surgical field decreased the hemodynamic effects of VAE, since $\mathrm{CO}_{2}$ emboli are more soluble and therefore better tolerated than air emboli. ${ }^{42}$

Matjasko et al reported a significantly higher incidence of VAE in children (62\%) compared with adults (23\%); however, a retrospective comparison between adults and children undergoing suboccipital craniotomy in the sitting position by Cucchiara et al showed a lower incidence of VAE in children (16 of 48) than in adult controls (22 of 48).43,44 Bithal et al similarly reported the incidence as $22 \%$ in children and $28 \%$ in adults out of a total of 430 patients ( 96 children and 334 adults) who underwent posterior fossa surgery in the sitting position. ${ }^{45}$ As per Gupta et al, since the sagittal sinus pressure remains positive in children even in the sitting position up to the age of 9 years, VAE was compared in children between the age range of above 2 years and below 9 years and in those above 9 years of age, but the incidence of VAE was found to be comparable ( $p=0.7)$ in the two groups. ${ }^{46}$

Gupta et al noted in their study of complications related to the sitting position during pediatric neurosurgery that intraoperative hemodynamic instability can occur unrelated to VAE. These were mainly bradycardia, tachycardia, and occasional ventricular premature beats, associated with hypotension or hypertension, due to tumor manipulation near the brainstem, and they resolved spontaneously after cessation of surgical stimulation. ${ }^{46}$ 
One of the advantages of the sitting position is decreased blood loss due to drainage of cerebral venous blood away from the operating site. Black et al reported that in adult patients, intraoperative blood loss and volume of blood transfused were significantly less in patients undergoing posterior fossa craniectomy in sitting position as compared with those operated in the horizontal position. ${ }^{47}$ Orliaquet et al compared perioperative complications in children undergoing posterior fossa surgery in sitting and prone positions and concluded that the volume of intraoperative blood transfusion was significantly larger in the prone position as compared with the sitting position. ${ }^{48}$

Schäfer et al analyzed the records of 799 patients between 1990 and 2009 and found that VAE tends to activate platelets, evoke thrombocytopenia, and platelet dysfunction. In patients who had VAE in this study, there was a significant decrease in mean platelet count, whereas in age-matched controls without VAE, the mean platelet count did not change. ${ }^{49}$

Rath et al, in their study of complications related to positioning in posterior fossa craniectomy, found that prolonged postoperative mechanical ventilation was seen more in the sitting position, probably due to brainstem handling. However, lower cranial nerve functions were preserved better in the sitting position $(p<0.05) .^{34}$

Postoperative pneumocephalus develops in the sitting position, and the incidence may reach $100 \%$. It is due to the intraoperative drainage of CSF, commonly via the subarachnoid drainage and the gravitational effect of sitting position, or residual air during closure of the dura. It has been attributed to the diminution of brain volume secondary to mannitol administration, hyperventilation, removal of the space-occupying mass, and contraction of intravascular blood volume due to intraoperative hemorrhage. ${ }^{26,45,47}$ Nitrous oxide, if used, should be discontinued 20 to 30 minutes before completion of the procedure; however, pneumocephalus can develop even without the use of nitrous oxide and may persist for weeks after surgery. ${ }^{26,50}$ Drainage of CSF during the surgery creates a negative ICP that entrains air. Lunsford et al in 1979 described this as a phenomenon analogous to the entry of air into an inverted pop bottle in which as the fluid pours out, air bubbles to the top. If the patient has coexisting hydrocephalus, the risk of pneumocephalus is increased. ${ }^{51}$ Tension pneumocephalus, though rare, is a life-threatening emergency and should be considered whenever a patient fails to recover as expected following posterior fossa surgery. Sachkova et al, in their study of predictors of ventricular tension pneumocephalus after posterior fossa surgery in the sitting position, found an incidence of $3.9 \%$ of tension pneumocephalus cases, which produced severe neurological deterioration and required an external ventricular drain (EVD) insertion. ${ }^{52}$ Presenting symptoms include delayed recovery, severe restlessness, deterioration of consciousness, and seizures. Management of tension pneumocephalus involves the urgent relief of the ICP. This is achieved with urgent neurosurgical interventions such as craniotomy, drilling of burr holes, needle aspiration, ventriculostomy placement, and closure of the dural defects.
Other complications include subdural, epidural, and remote intraparenchymal hematomas..$^{53}$ Neck flexion and head tilt in the sitting position may cause acute parotitis. ${ }^{54}$ Piriformis syndrome may occur due to compression of sciatic nerve. Recurrent laryngeal nerve palsy and tension pneumoventricle are also associated with sitting position..$^{55}$

\section{Conclusion}

Ideal patient positioning involves balancing surgical comfort against risks related to the patient. The sitting position offers many benefits; however, we should be aware of the physiological changes and complications that may occur during positioning. A thorough risk-benefit ratio based on the patient's physical status and surgical implications need to be discussed among the team members and the patient. A strict sitting position protocol should be in place so that specific difficult surgical access cases may not be denied the technical advantages of sitting craniotomy. With thorough preoperative anesthetic evaluation, use of advanced intraoperative monitoring, and meticulous surgical techniques, the sitting position can be safely used in indicated cases.

\section{Conflict of Interest}

None declared.

\section{References}

1 Porter JM, Pidgeon C, Cunningham AJ. The sitting position in neurosurgery: a critical appraisal. $\mathrm{Br} \mathrm{J}$ Anaesth 1999;82(1):117-128

2 Sala F, Gallo P, Tramontano V, Gerosa M. Intraoperative neurophysiological monitoring in posterior fossa surgery. In: Özek MM, Cinalli G, Maixner W, Sainte-Rose C, eds. Posterior Fossa Tumors in Children Switzerland: Springer International Publishing;2015:239-262

3 Heymanns V, Jung S, Tallo A, Cheko A, Alyeldien A, et al. Experiences with the sitting position in posterior fossa surgery in 310 patients. J Neurol Disord 2015;S1:002

4 De Martel T. Surgical treatment of cerebral tumors: technical considerations. Surg Gynecol Obstet 1931;52:381-385

5 Frazier $\mathrm{CH}$, Gardner WI. The radical operation for the relief of trigeminal neuralgia. Surg Gynecol Obstet 1928;47:73-77

6 Slbin MS, Babinski M, Maroon JC, Jannetta PJ. Anesthetic management of posterior fossa surgery in the sitting position. Acta Anaesthesiol Scand 1976;20(2):117-128

7 Kwee MM, Ho YH, Rozen WM. The prone position during surgery and its complications: a systematic review and evidence-based guidelines. Int Surg 2015;100(2):292-303

8 Klein J, Juratli TA, Weise M, Schackert G. A systematic review of the semi-sitting position in neurosurgical patients with patent foramen ovale: how frequent is paradoxical embolism? World Neurosurg 2018;115:196-200

9 Fathi AR, Eshtehardi P, Meier B. Patent foramen ovale and neurosurgery in sitting position: a systematic review. $\mathrm{Br} \mathrm{J}$ Anaesth 2009;102(5):588-596

10 Caputi L, Carriero MR, Falcone C, et al. Transcranial Doppler and transesophageal echocardiography: comparison of both techniques and prospective clinical relevance of transcranial Doppler in patent foramen ovale detection. J Stroke Cerebrovasc Dis 2009;18(5):343-348 
11 Sivanaser V, Manninen P. Preoperative assessment of adult patients for intracranial surgery [review]. Anesthesiol Res Pract 2010; 2010

12 Hambidge O, John R. Anaesthesia for neurosurgery. Anaesth Intensive Care Med 2016;17(12):625-629

13 Chui J, Mariappan R, Mehta J, Manninen P, Venkatraghavan L. Comparison of propofol and volatile agents for maintenance of anesthesia during elective craniotomy procedures: systematic review and meta-analysis. Can J Anaesth 2014;61(4):347-356

14 Bastola P, Bhagat H, Wig J. Comparative evaluation of propofol, sevoflurane and desflurane for neuroanaesthesia: a prospective randomised study in patients undergoing elective supratentorial craniotomy. Indian J Anaesth 2015;59(5):287-294

15 Losasso TJ, Muzzi DA, Dietz NM, Cucchiara RF. Fifty percent nitrous oxide does not increase the risk of venous air embolism in neurosurgical patients operated upon in the sitting position. Anesthesiology 1992;77(1):21-30

16 Losasso TJ, Black S, Muzzi DA, Michenfelder JD, Cucchiara RF. Detection and hemodynamic consequences of venous air embolism. Does nitrous oxide make a difference? Anesthesiology 1992;77(1):148-152

17 Voorhies RM, Fraser RA, Van Poznak A. Prevention of air embolism with positive end expiratory pressure. Neurosurgery 1983;12(5):503-506

18 Giebler R, Kollenberg B, Pohlen G, Peters J. Effect of positive end-expiratory pressure on the incidence of venous air embolism and on the cardiovascular response to the sitting position during neurosurgery. Br J Anaesth 1998;80(1):30-35

19 Dilmen OK, Akcil EF, Tureci E, et al. Neurosurgery in the sitting position: retrospective analysis of 692 adult and pediatric cases. Turkish Neurosurgery 2011;21(4):634-640

20 Gildenberg PL, O'Brien RP, Britt WJ, Frost EA. The efficacy of Doppler monitoring for the detection of venous air embolism. J Neurosurg 1981;54(1):75-78

21 Giraldo M, Lopera LM, Miguel A M. Essay: venous air embolism in neurosurgery. Rev Colomb Anestesiol 2015;43(S1):40-44

22 Feigl GC, Decker K, Wurms M, et al. Neurosurgical procedures in the semisitting position: evaluation of the risk of paradoxical venous air embolism in patients with a patent foramen ovale. World Neurosurg 2014;81(1):159-164

23 Pandia MP, Bithal PK, Dash HH, Chaturvedi A. Comparative incidence of cardiovascular changes during venous air embolism as detected by transesophageal echocardiography alone or in combination with end tidal carbon dioxide tension monitoring. J Clin Neurosci 2011;18(9):1206-1209

24 Heroabadi A, Babakhani B, Azimaraghi O, et al. Cerebral oxygen monitoring: an observational prospective study on seated position neurosurgical procedures. Arch Neurosci 2017;4(4):e56123

25 Schramm P, Tzanova I, Hagen F, et al. Cerebral oxygen saturation and cardiac output during anaesthesia in sitting position for neurosurgical procedures: a prospective observational study. Br J Anaesth 2016;117(4):482-488

26 Rozet I, Vavilala MS. Risks and benefits of patient positioning during neurosurgical care. Anesthesiol Clin 2007;25(3):631-653,x

27 Hagan K, Gottumukkala V, Surgical positioning: physiology and perioperative implications. In: Longnecker DE, Brown D, Newman M, Zapol WM, eds. Anesthesiology. 3rd ed. Chap 23; New York, NY: McGraw-Hill; 2017:316-325

28 Srivastava S. The sitting position for neurosurgery: a bane or a boon. Neurol India 2018;66(1):223-225

29 Ranjith M, Bidkar PU, Narmadalakshmi K, Talawar PR. Effects of crystalloid preloading $(20 \mathrm{ml} / \mathrm{kg}$ ) on hemodynamics in relation to postural changes in patients undergoing neurosurgical procedures in sitting position. J Neurosci Rural Pract 2018;9(1):80-85
30 Dohn DF, Gardner WJ. The antigravity suit (G-suit) in surgery; control of blood pressure in the sitting position and in hypotensive anesthesia. J Am Med Assoc 1956;162(4):274-276

31 Meyer PG, Cuttaree H, Charron B. Jarreau MM, Perie AC, Sainte-Rose C. Prevention of venous air embolism in paediatric neurosurgical procedures performed in the sitting position by combined use of MAST suit and PEEP. Br J Anaesth 1994;73(6):795-800

32 Martin JT. Neuroanesthetic adjuncts for surgery in the sitting position. I. Introduction and basic equipment. Anesth Analg 1970;49(4):577-587

33 Abd El-Ghani Wael MA. Review of the basic principles of semisitting position in neurosurgery. AAMJ 2015;13 (4, Suppl 2): $21-25$

34 Rath GP, Bithal PK, Chaturvedi A, Dash HH. Complications related to positioning in posterior fossa craniectomy. J Clin Neurosci 2007;14(6):520-525

35 Deinsberger W, Christophis P, Jödicke A, Heesen M, Böker DK. Somatosensory evoked potential monitoring during positioning of the patient for posterior fossa surgery in the semisitting position. Neurosurgery 1998;43 (1):36-40,discussion40-42

36 American Society of Anesthesiologists. Task force on the prevention of perioperative peripheral neuropathies: practice advisory for the prevention of perioperative peripheral neuropathies. Anesthesiology 2000;92:1168-1182

37 Mirski MA, Lele AV, Fitzsimmons L, Toung TJK. Diagnosis and treatment of vascular air embolism. Anesthesiology 2007;106(1):164-177

38 Ganslandt O, Merkel A, Schmitt $\mathrm{H}$, et al. The sitting position in neurosurgery: indications, complications and results. a single institution experience of 600 cases. Acta Neurochir (Wien) 2013;155(10):1887-1893

39 Mammoto T, Hayashi Y, Ohnishi Y, Kuro M. Incidence of venous and paradoxical air embolism in neurosurgical patients in the sitting position: detection by transesophageal echocardiography. Acta Anaesthesiol Scand 1998;42(6):643-647

40 Harrison EA, Mackersie A, McEwan A, Facer E. The sitting position for neurosurgery in children: a review of 16 years' experience. Br J Anaesth 2002;88(1):12-17

41 Eckle VS, Neumann B, Greiner TO, Wendel HP, Grasshoff C. Intrajugular balloon catheter reduces air embolism in vitro and in vivo. Br J Anaesth 2015;114(6):973-978

42 Longatti P, Marton E, Feletti A, Falzarano M, Canova G, Sorbara C. Carbon dioxide field flooding reduces the hemodynamic effects of venous air embolism occurring in the sitting position. Childs Nerv Syst 2015;31(8):1321-1326

43 Matjasko J, Petrozza P, Cohen M, Steinberg P. Anesthesia and surgery in the seated position: analysis of 554 cases. Neurosurgery 1985;17(5):695-702

44 Cucchiara RF, Bowers B. Air embolism in children undergoing suboccipital craniotomy. Anesthesiology 1982;57(4):338-339

45 Bithal PK, Pandia MP, Dash HH, Chouhan RS, Mohanty B, Padhy N. Comparative incidence of venous air embolism and associated hypotension in adults and children operated for neurosurgery in the sitting position. Eur J Anaesthesiol 2004;21(7):517-522

46 Gupta P, Rath GP, Prabhakar H, Bithal PK. Complications related to sitting position during pediatric neurosurgery: an institutional experience and review of literature. Neurol India 2018;66(1):217-222

47 Black S, Ockert DB, Oliver WC Jr, Cucchiara RF. Outcome following posterior fossa craniectomy in patients in the sitting or horizontal positions. Anesthesiology 1988;69(1):49-56

48 Orliaguet GA, Hanafi M, Meyer PG, et al. Is the sitting or the prone position best for surgery for posterior fossa tumours in children? Paediatr Anaesth 2001;11(5):541-547 
49 Schäfer ST, Sandalcioglu IE, Stegen B, Neumann A, Asgari S, Peters J. Venous air embolism during semi-sitting craniotomy evokes thrombocytopenia. Anaesthesia 2011;66(1):25-30

50 Duke DA, Lynch JJ, Harner SG, Faust RJ, Ebersold MJ. Venous air embolism in sitting and supine patients undergoing vestibular schwannoma resection. Neurosurgery 1998;42(6):1282-1286

51 Lunsford LD, Maroon JC, Sheptak PE, Albin MS. Subdural tension pneumocephalus. Report of two cases. J Neurosurg 1979;50(4):525-527

52 Sachkova A, Schemmerling T, Goldberg M, et al. Predictors of ventricular tension pneumocephalus after posterior fossa surgery in the sitting position. Acta Neurochir (Wien) 2018;160(3):525-538

53 Basma J, Nguyen V, Sorenson J, Intracranial procedures in the supine, sitting and semi-sitting positions. In: Arthur A, Foley $\mathrm{K}$, Hamm C, eds. Perioperative Considerations and Positioning for Neurosurgical Procedures. Springer; 2018:83-100

54 Berker M, Sahin A, Aypar U, Ozgen T. Acute parotitis following sitting position neurosurgical procedures: review of five cases. J Neurosurg Anesthesiol 2004;16(1):29-31

55 Radhziah S, Lee CK, Ng I. Tension pneumoventricle. J Clin Neurosci 2006;13(8):881-883 